\title{
Stability of $p$-Cycles under Dynamic Traffic
}

\author{
Brigitte Jaumard \\ CIISE, Concordia University \\ Montreal (Qc), H3G 1M8- Canada \\ Email: bjaumard@ciise.concordia.ca
}

\author{
Ammar Metnani \\ DIRO, Université de Montréal \\ Montreal (Qc), H3C 3J7- Canada \\ Email: metnania@iro.umontreal.ca
}

\begin{abstract}
Cycles offer a protection that corresponds to an efficient pre-configured and pre-cross-connected protection scheme. Thereafter, we study the stability and the efficient reconfiguration of $p$-cycles in the context of dynamic asymmetric traffic. The literature acknowledges it as a difficult problem, but which is of paramount importance in the selection of $p$-cycles as a protection scheme in Intelligent Optical Networks (IONs).

We investigate two $p$-cycle updating models which differ mainly by their objective criteria. The first objective is the classical one, aiming at minimizing the spare capacity. The second one is new and corresponds to the number of pre-crossconnections that needs to be set/reset after each traffic variation, while attempting to maximize the re-use of previously established pre-cross-connection settings in spite of the modifications of the $p$-cycles. Note that the second model needs to explicitly include wavelength continuity constraints and is therefore slightly more complex. Results show that $(i)$ the proposed ILP tools are scalable, and (ii) while $p$-cycles appear not to be very stable with the first criterion, they emerge as highly stable with the second criterion.
\end{abstract}

\section{INTRODUCTION}

While $p$-cycles have been studied a lot in the context of static and symmetrical traffic, traffic demand is usually changing dynamically and becoming more asymmetrical. Consequently working paths need to be set/reset dynamically and protection must be adapted accordingly. We propose to investigate further the $p$-cycle design problem in such a context, as few papers have yet been devoted to it.

$p$-Cycles are gaining in popularity among the protection schemes for WDM (Wavelength Division Multiplexing) networks due to their pre-configuration and pre-cross-connectivity features. In case of a link failure, i.e., the most frequent failure, only the two endpoints of the failing fiber link need to be reconfigured.

Most studies on $p$-cycles consider the minimization of the protection capacity without worrying about the wavelength continuity constraint which is underlying the particular and advantageous fully pre cross-connected feature of $p$-cycles. For this reason, we will revisit the stability of $p$-cycles assuming we minimize the number of OXC (Optical Cross Connect) port settings under the wavelength continuity assumption.

We next review the relevant papers: firstly, dynamic $p$-cycle design and secondly, wavelength assignment in $p$-cycles.

As for the static $p$-cycle problem, two approaches have been explored for the design and management of dynamic $p$-cycles. The first one is the off-line approach, which consists in offline generating either the set of all possible $p$-cycles [1], [2], or a restricted set of promising candidate $p$-cycles [3]. The second approach, i.e., the on-line one that we will adopt in this paper, consists in generating one (few) $p$-cycle(s) at a time, and adding it only if it contributes to the improvement of the current solution. While several authors have successfully used Integer Linear Programs (ILPs) in studies under static traffic, it has been strongly questioned in the case of dynamic traffic for scalability reasons, see, e.g., [2]. We believe there is a misunderstanding on the usefulness of ILP tools. Indeed, their strength is often under evaluated, as their use is frequently not pushed to their limit, and not sufficiently combined with the nowadays available large scale optimization tools.

Previous studies on dynamic $p$-cycles include the work of Zhong et al. [2] and of Ruan et al. [3]. Both studies use the grade of service metric in order to evaluate the performance of their strategies. However, the transport capacity is often set to an arbitrary value with, e.g., a dedicated uniform share of $50 \%$ for protection on all the links, even if it is well known, that even under a uniform distribution of the traffic between all node pairs, not all links are uniformly used depending on the topology of the network under study.

Very few studies have investigated the issue of wavelength assignment in the context of either static or dynamic $p$ cycles. In Eshoul and Mouftah [4], two 0-1 ILP models are proposed, but as they are not scalable, heuristic solutions are used instead. An extensive comparison of several precross-connected protection architectures is made in Grue et al. [5] that includes $p$-cycles under a wavelength assignment assumption, but with very little details on the exactness of the ILP models and the accuracy of their solutions.

The paper is organized as follows. In the next section, we recall the ILP model [6] with the spare capacity objective and develop a new one with the pre-cross-connection objective for the updating of $p$-cycles under dynamic traffic. As the second model needs to explicitly include wavelength continuity constraints, it is therefore slightly more complex than the first one. We next explain how to solve the models in Section III. In Section IV, we first examine the quality and the characteristics of the solutions, and then we evaluate the comparative performances of the two models in order to provide some new answers on the controversial question of the stability of $p$-cycles under dynamic traffic. Conclusions are drawn in the last section. 


\section{II. $p$-CyCle PRotection DeSign Under DyNAmiC TRAFFIC}

\section{A. Generalities}

We investigate the problem of the updating of $p$-cycles under dynamic traffic, using two different objectives. The first one is the classical objective that has been used in the literature so far, i.e., the minimization of the spare capacity (or protection capacity). The second one, which might be more meaningful for network designers/managers, corresponds to the number of $\mathrm{OXC}$ ports, or required port reconfigurations in the context of a dynamic traffic.

Traffic instances are described by a set of requests where, for each request, we are given its source, its destination and its bandwidth requirement. Note that the traffic is assumed to be directed as it appears a more meaningful hypothesis, and as a consequence, as we will see in Section II-B, the benefit of straddling links in $p$-cycles is reduced. We will assume that the routing of the working paths is given, and that it has been performed, e.g., using shortest path routing. Using the routing of the working paths, we then deduce the amount of bandwidth that needs to be protected on each directed link.

In this study, as in [7], we propose to examine dynamic provisioning with the framework of small-batch provisioning under asymmetric traffic. Indeed, even if a wide range of applications may be envisioned to require on-demand connection provisioning, it seems reasonable that a delay in the range of few seconds up to few minutes, depending on the applications, can be reasonably tolerated between connection request and setup. In the context of a core network, we are dealing with the establishment of lightpaths that can convey up to $10 \mathrm{Gbs}$ or even $40 \mathrm{Gbs}$, and costs thousand of dollars to use. We can think of routers making the request for an additional lightpath on the basis of observed trends, slightly before the added capacity is fully needed, see [7].

\section{B. Mathematical Models}

We first define the notations, parameters and variables, and then the mathematical models in the two subsequent subsections.

1) Notations: We represent the optical network by a directed graph $G=(V, L)$ where $V$ represents the set of optical nodes, indexed by $v$, and where $L$ is associated with the set of optical directional links, indexed by $\ell$. Each link is characterized by its transport capacity (or equivalently the number of wavelengths or the number of bandwidth units it can support) $\bar{c}_{\ell}$ and its $\operatorname{COST}_{\ell}$. Let $\Lambda$ be the set of available wavelengths (the same number for each link), with generic index $\lambda$ and $W=|\Lambda|$.

Let $T$ be the overall set of time periods, indexed by $t$, where each time period is associated with a batch of some add/drop connection requests. We develop the mathematical model at an arbitrary period, say $t$, taking into account the new incoming and the terminating requests, as well as the traffic legacy, i.e., the set of ongoing protected requests. Parameter $w_{\ell}^{t}$ is the overall number of bandwidth units that need to be protected on link $\ell$ during time period $t$. Parameter $y_{c}^{t-1}$ represents the overall number of required copies of $p$-cycle $c$ in order to protect the ongoing requests that have been granted during the previous time periods.

Let $\mathcal{C}$ be the set of all potential cycles, indexed by $c$ and $\mathcal{C}^{\lambda}$ be the set of all potential cycles with wavelength $\lambda$. We denote by $\mathcal{C}^{<t}$ the set of $p$-cycles which have established during the previous time periods. We use two sets of parameters: the first one corresponds to the binary parameters $a_{\ell}^{c}$ such that $a_{\ell}^{c}=1$ if link $\ell \in L$ is an on-cycle link of $p$-cycle $c$ and 0 otherwise. The second set corresponds to the binary parameters $s_{\ell}^{c}$ such that $s_{\ell}^{c}=1$ if link $\ell$ is a straddling one with respect to $p$-cycle $c$ and 0 otherwise.

We denote by $c_{\ell}^{R}$ the residual transport capacity of link $\ell$ once the routing of the working paths has been completed: $c_{\ell}^{R}=\bar{c}_{\ell}-w_{\ell}$.

We next introduce the sets of variables. The first set of variables, represented by vector $y^{t}=\left(y_{c}^{t}\right) \in \mathrm{Z}_{+}^{|\mathcal{C}|}$ is such that $y_{c}^{t}$ is the number of new required copies of $p$-cycle $c$ during time period $t$, in order to grant all new incoming requests (beware that definitions of $y_{c}^{t}$ and $y_{c}^{t-1}$ differs in the scope of connection requests they encompass).

Depending on the applications, longer end-to-end delays can be acceptable. We exploit this tolerance in order to briefly disturb the protection of a limited number of tolerant connections, in order to increase the number of protected granted connections (transport capacity constraints may limit the number of granted requests) and minimize the protection cost (whether it is bandwidth consumption or equipment cost). For this reason, we allow the re-arrangement of a limited number $(\bar{z})$ of copies of $p$-cycles. The second set of variables, $z=\left(z_{c}^{t-1}\right) \in \mathrm{Z}_{+}^{|\mathcal{C}|}$ is such that $z_{c}^{t-1}$ is the number of dismantled copies of $p$-cycle $c$ during period $t$ in order to grant more efficiently the new incoming requests, and allow accordingly some $p$-cycle re-arrangement. Consequently, $y_{c}^{t-1}=z_{c}^{t-1}+x_{c}^{t-1}$, where $x_{c}^{t-1}$, the variables of the third set are such that $x_{c}^{t-1}$ is equal to the number of maintained copies of $p$-cycles from the previous time periods for protecting the ongoing requests. Note that a $p$-cycle can be dismantled either because it is no more useful in order to protect some working bandwidth, or because it is more advantageous to replace it by another more bandwidth efficient $p$-cycle(s).

2) Model 1: Updating p-cycles with minimum bandwidth cost: The first model, where the objective, to be minimized, evaluates the link cost, can be written as follows:

$$
\min f_{1}^{\text {ов }}=\sum_{\ell \in L} \operatorname{cosT}_{\ell}\left(\sum_{c \in \mathcal{C}} a_{\ell}^{c} y_{c}^{t}-\sum_{c \in \mathcal{C}<t} a_{\ell}^{c} z_{c}^{t-1}\right)
$$

subject to:

$$
\begin{aligned}
& \sum_{c \in \mathcal{C}}\left(a_{\ell}^{c}+s_{\ell}^{c}\right) y_{c}^{t}-\sum_{c \in \mathcal{C}<t}\left(a_{\ell}^{c}+s_{\ell}^{c}\right) z_{c}^{t-1} \geq \\
& w_{\ell}-\sum_{c \in \mathcal{C}<t}\left(a_{\ell}^{c}+s_{\ell}^{c}\right) x_{c}^{t-1} \quad \ell \in L
\end{aligned}
$$




$$
\begin{array}{ll}
\sum_{c \in \mathcal{C}} a_{\ell}^{c} y_{c}^{t}-\sum_{c \in \mathcal{C}<t} a_{\ell}^{c} z_{c}^{t-1} \leq c_{\ell}^{R}-\sum_{c \in \mathcal{C}<t} a_{\ell}^{c} x_{c}^{t-1} & \ell \in L \\
\sum_{c \in \mathcal{C}<t} z_{c}^{t-1} \leq \bar{z} & \\
y_{c}^{t}, x_{c}^{t-1}, z_{c}^{t-1} \in \mathrm{Z}^{+} & c \in \mathcal{C}
\end{array}
$$

Constraints (1) ensure that the overall working traffic on each link is protected. Constraints (2) ensure that the overall protection capacity does not exceed the residual capacity on each link, while constraint (3) ensures that the number of dismantled copies does not exceed a given threshold. Note that, contrarily to what happens in the models under the assumption of undirected traffic, the straddling links can be only protected by one of the half of the $p$-cycle they are associated with.

3) Model 2: Updating p-cycles with minimum port cost: The second model aims at evaluating the port cost, and the number of OXC ports that need to be reset when updating/modifying $p$-cycles following some traffic variations, subject to the wavelength continuity constraints. Those last constraints are expressed throughout the definition of a cycle configuration: each configuration is in one to one correspondence with a wavelength, with $\mathcal{C}=\bigcup_{\lambda \in \Lambda} \mathcal{C}^{\lambda}$. The objective function, to be minimized, evaluates the number of $\mathrm{OXC}$ port reconfigurations:

$$
\begin{aligned}
f_{2}^{\mathrm{OBJ}=\min } & \sum_{\lambda \in \Lambda} \sum_{c \in \mathcal{C}^{\lambda}} \sum_{\ell=\left(v, v^{\prime}\right) \in L} \sum_{\ell^{\prime} \in w^{+}\left(v^{\prime}\right)} \\
& (\underbrace{a_{\ell}^{c} a_{\ell^{\prime}}^{c}}_{\text {Part 1 }}-\underbrace{\sum_{c^{\prime} \in \mathcal{C} \mathcal{C}_{\lambda}^{<t}}\left(a_{\ell}^{c} a_{\ell^{\prime}}^{c}\right)\left(a_{\ell}^{c^{\prime}} a_{\ell^{\prime}}^{c^{\prime}}\right) z_{c^{\prime}}^{t-1}}_{\text {Part 2 }}) y_{c}^{t}
\end{aligned}
$$

On each copy of a $p$-cycle, a node pre-cross-connection involves two ports: one input port on the incoming on-cycle link and one output port on the outgoing on-cycle link, with both links assigned the same wavelength, say $\lambda$, for the $p$-cycle copy, or embedded in the same configuration $c$ if we deal with wavelength configuration. Such a pre-cross-connection, within the context of a wavelength configuration (or $p$-cycle) $c$, is associated with the product $a_{\ell}^{c} a_{\ell^{\prime}}^{c}$, with parameters $a_{\ell}^{c}$ and $a_{\ell^{\prime}}^{c}$ as defined in Subsection II-B1. It is equal to 1 if there is an operational pre-cross-connection with incoming link $\ell$ and outgoing link $\ell^{\prime}$ in configuration $c, 0$ otherwise.

At time period $t-1$, a pre-cross-connection $a_{\ell}^{c} a_{\ell^{\prime}}^{c}$ on $p$ cycle $c \in \mathcal{C}^{\lambda}$ can be reused during time period $t$ in a $p$ cycle $c^{\prime} \in \mathcal{C}^{\lambda^{\prime}}$, if and only if $p$-cycle $c$ is dismantled and $\lambda=\lambda^{\prime}$. An example of such a case is illustrated in Figure 1 where we show the evolution of a $p$-cycle from period $t-1$ to period $t$. While $p$-cycle $c_{1}$, in operation during period $t-1$, is dismantled one period later, the pre-cross-connections at nodes $v_{4}$ and $v_{5}$ can be preserved and reused in $p$-cycle $c_{2}$ at time period $t$. The pre-cross-connection cost of $p$-cycle $c_{2}$ is therefore reduced to 3 (instead of 5 if we did care re-using the previously established pre-cross-connections).

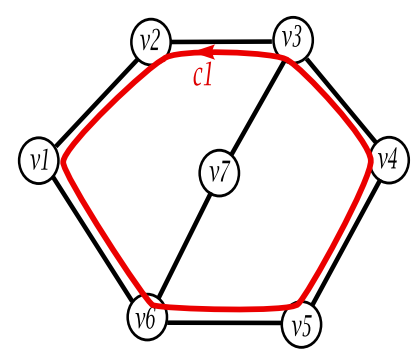

(a) Time period $t-1$

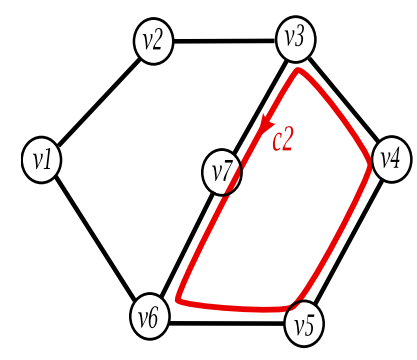

(b) Time period $t$
Fig. 1. Reuse of pre-cross-connections

Coming back to the analytical expression of the objective function, the term called "Part1" counts the number of precross-connections of the $c$ configuration, and subtract from it (term "Part2") those which have been already set in eliminated $p$-cycles and can therefore be reused.

The set of constraints follows and is very similar to the one of Model 1, with the additional concern about the wavelength continuity constraints in order to be able to compute accurately the number of pre-cross-connections, see constraints (8).

$$
\begin{aligned}
& \sum_{c \in \mathcal{C}}\left(a_{\ell}^{c}+s_{\ell}^{c}\right) y_{c}^{t}-\sum_{c \in \mathcal{C}<t}\left(a_{\ell}^{c}+s_{\ell}^{c}\right) z_{c}^{t-1} \geq \\
& w_{\ell}-\sum_{c \in \mathcal{C}<t}\left(a_{\ell}^{c}+s_{\ell}^{c}\right) x_{c}^{t-1} \quad l \in L \\
& \sum_{c \in \mathcal{C}} a_{\ell}^{c} y_{c}^{t}-\sum_{c \in \mathcal{C}<t} a_{\ell}^{c} z_{c}^{t-1} \leq c_{\ell}^{R}-\sum_{c \in \mathcal{C}<t} a_{\ell}^{c} x_{c}^{t-1} \quad \ell \in L \\
& \sum_{c \in \mathcal{C}^{\lambda}} a_{\ell}^{c} y_{c}^{t}-\sum_{c \in \mathcal{C}_{\lambda}^{<t}} a_{\ell}^{c} z_{c}^{t-1} \leq \\
& 1-\sum_{c \in \mathcal{C}_{\lambda}^{<t}} a_{\ell}^{c} x_{c}^{t-1} \quad \ell \in L, \lambda \in \Lambda \\
& \sum_{c \in \mathcal{C}<t} z_{c}^{t-1} \leq \bar{z} \\
& y_{c}^{t}, x_{c}^{t-1}, z_{c}^{t-1} \in\{0,1\} \\
&
\end{aligned}
$$

Constraints (6) ensure that the overall working traffic on each link is protected. Constraints (7) ensure that the overall protection capacity does not exceed the residual capacity on each link, while constraint (9) ensures that the number of dismantled copies does not exceed a given threshold.

\section{Solving The Mathematical Models}

We discuss here how to solve efficiently the ILP models developed in the previous section.

\section{A. Large Scale Optimization Tools}

As for the efficient computation of $p$-cycles in a static model, we have different choices for solving the models developed in the previous section. Obviously, enumerating explicitly all possible cycles will not lead to a scalable solution 
scheme. Therefore, we can either develop a heuristic in order to generate explicitly only the most promising cycles as in [8], assuming we can define a good metric to identify the most promising, or we can generate implicitly all potential cycles with the use of the column generation techniques combined with either a heuristic or a branch-and-price for solving the ILP part as in, e.g., [9]. We propose to go in this last direction with the use of column generation techniques and to devise how to derive a near optimal ILP solution once we have computed the optimal LP solution.

Column generation techniques involve the decomposition of the original problem into a master problem corresponding to the models developed in Section II-B and a pricing problem corresponding to the on-line generation of a new cycle which is guaranteed to improve the current LP solution. The improvement guarantee is obtained thanks to the identification of a cycle with a negative reduced cost, where the reduced cost, an LP indicator, is defined as follows, for Model 1:

$$
\overline{\operatorname{COST}}_{c}=\operatorname{cosT}_{c}-\sum_{\ell \in L} u_{\ell}^{1}\left(a_{\ell}^{c}+s_{\ell}^{c}\right)+\sum_{\ell \in L} u_{\ell}^{2} a_{\ell}^{c},
$$

where $\operatorname{CosT}_{c}$ is the unit cost of cycle $c$, given by $\operatorname{COST}_{c}=$ $\sum_{\ell \in L} a_{\ell}^{c} \operatorname{CosT}_{\ell}$, and where $u_{\ell}^{1} \geq 0$ and $u_{\ell}^{2} \leq 0$ are the dual variables of constraints (1) and (2) respectively.

At each iteration of the column generation algorithm, the pricing problem looks for the cycle with a negative reduced cost (but not necessarily the most negative one). Description of the set of constraints of the pricing problem can be easily deduced from the pricing problem arising in the column generation formulations and solutions of the static $p$-cycle problem, see, e.g., [10]. If such a cycle is found, it is added to the master problem, which is solved again optimally. New dual variable values are generated and again thrown in the pricing problem, to generate another cycle. This process is repeated until no cycle with a negative reduced cost is found, then we can claim that the current solution is optimal. Let us denote by $f_{\mathrm{LP}}^{\star}$ the optimal solution of the linear relaxation of say the mathematical formulation of Model 1. Very often, $f_{\mathrm{LP}}^{\star}$ is not associated with an integer vector $y^{\star}$ but only provides a lower bound, denoted by $\tilde{f}_{\mathrm{LP}}$, on the optimal integer value, say $f_{\mathrm{ILP}}^{\star}$. Solving the ILP deduced from the constraint matrix derived from the set of columns generated in order to obtain $z_{\mathrm{LP}}^{\star}$ leads to a feasible integer solution whose value, $\tilde{f}_{\mathrm{LP}}$, is often near optimal, i.e., such that the optimality gap $\tilde{f}_{\mathrm{LLP}}-f_{\mathrm{LP}}^{\star}$ is often very small, i.e., less than $1 \%$. In the context of the present study, we derive an ILP solution using a rounding off method as in [10].

\section{B. Particularities of Model 2}

1) Nonlinearities of the objective function: As one can observe, the objective function of Model 2 is nonlinear due to the presence of products of variables $y_{c}^{t} z_{c^{\prime}}^{t-1}$. However, observe first the column generation only involve the $y_{c}^{t}$ variables, the number of variables $z_{c}^{t-1}$ remains identical during the solution process of the column generation algorithm (for a given time period, but of course varies from one time period to the next).
Secondly, it is indeed easy to overcome the nonlinearities using the following remark. The cost of all disturbed $p$-cycles is equal to 0 : if we eliminate one $p$-cycle, there is nothing to gain except if we re-use some of its pre-cross-connections. Each time we solve the pricing problem, we generate a cycle using the reduced cost where the cost is evaluated with the number of required pre-cross-connections for establishing the generated cycle (part 1 in (5)), from which we subtract the re-usable pre-cross-connections of the disturbed $p$-cycles (part 2 in (5)).

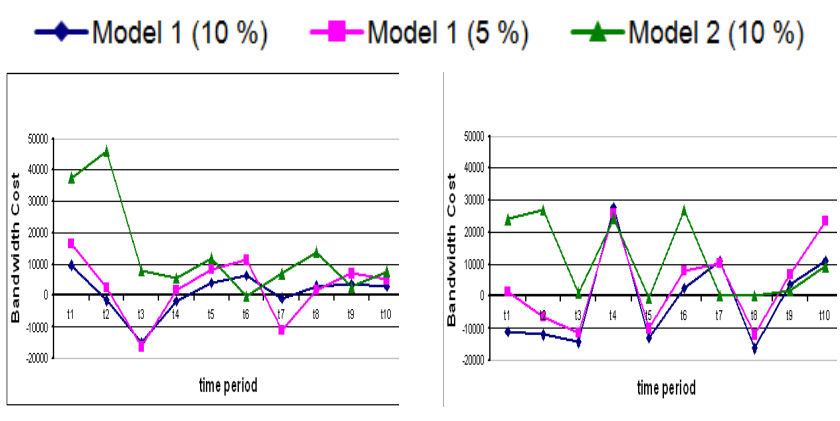

(b) COST239 network
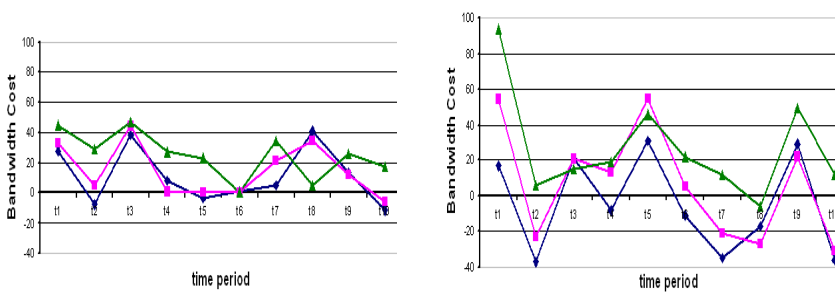

(c) NY network

Fig. 2. Variations of the $p$-Cycle bandwidth costs in Models $1 \& 2$

2) Building an initial solution for Model 2: At the outset time period, the objective is to minimize the number of precross-connections which are required in order to make all the $p$-cycles operational. The expression of both the objective and of the constraints are simplified and can be written as follows:

$$
\begin{array}{ll}
\min _{2}^{\text {овл }(t=0)}=\sum_{\ell \in L} \sum_{c \in \mathcal{C}} a_{\ell}^{c} y_{c}^{t} & \\
\sum_{c \in \mathcal{C}}\left(a_{\ell}^{c}+s_{\ell}^{c}\right) y_{c}^{t} \geq w_{\ell} & \ell \in L \\
\sum_{c \in \mathcal{C}} a_{\ell}^{c} y_{c}^{t} \leq W & \\
y_{c}^{t} \in \mathrm{Z}^{+} & c \in L
\end{array}
$$

The ILP solution of the above model provides the number of copies $y_{c}^{t}$ of each selected $p$-cycle configuration. In order to be able to solve Model 2 in the subsequent time periods, we need to associate a wavelength with each copy of a $p$ cycle, while minimizing the number of required wavelengths. This corresponds to a simple wavelength assignment problem 
that we decided to solve using a reformulation of it as a graph coloring problem on an undirected conflict graph $G_{c}=\left(V_{c}, L_{c}\right)$, where each node $v \in V_{c}$ of the conflict graph is associated with one $p$-cycle copy. An edge exists between two nodes of the conflict graph if the two $p$-cycle copies associated with the nodes share a link. We used the classical and well known DSATUR heuristic (Brélaz [11]) to solve the graph coloring problem.
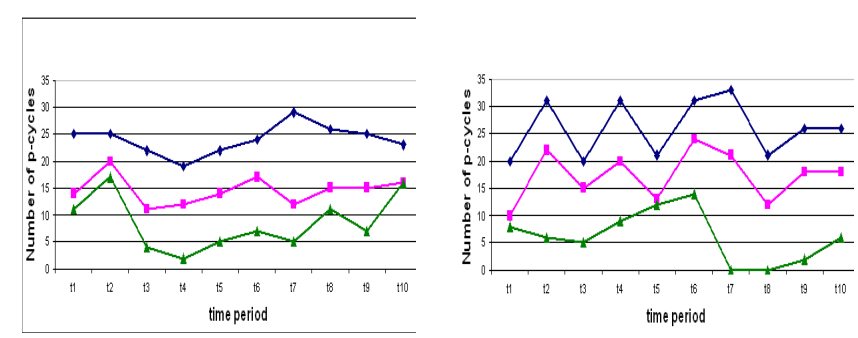

(a) COST239 network
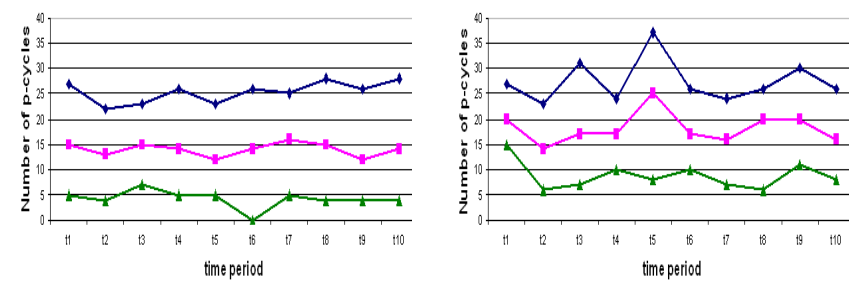

(b) NY network

Fig. 3. Stability of $p$-cycles (same legend as in Figure 2)

\section{Computational Results}

We first describe the data instances and next the various experiments we conducted with the two models. For each objective (or model), we evaluate the solutions, i.e., protection schemes, of the two models using the two metrics induced by the two objectives, i.e., the protection bandwidth requirements (even if not directly optimized in Model 2) and the number of new pre-cross-connections that need to be established after each traffic variation (even if not directly optimized in Model 1).

\section{A. Network and Traffic Instances}

We use two network instances, the COST239 network (11 nodes, 52 directed links, link cost as in [12]), and the NY network (16 nodes, 98 directed links, unit link cost).

We assume that, at the outset, we already have 2,000 established request connections, for all instances. At each time period, some connections are torn down and new incoming ones are established. We consider two different traffic scenarios, each with a stable overall traffic, but with different turn over ratios where the turn over ratio is defined by the ratio

$$
\frac{\max \{\# \text { ADD, } \# \text { DROP }\}}{\# \text { original connection requests }} \text {. }
$$

In the case of stable traffic, number of incoming requests is equal to the number of torn down ones. The first traffic scenario has a turn over ratio of $10 \%$, and the second one 30 $\%$.

Programs were run on a dual core AMD Opteron machine (2.5 Ghz, 16Gb RAM), using the CPLEX package (release 10).
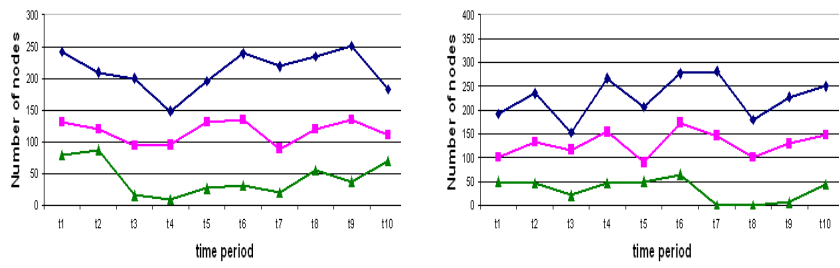

(a) COST239 network
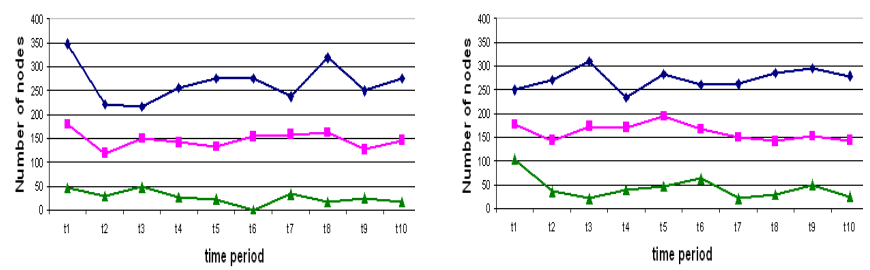

(b) NY network

Fig. 4. Re-using pre-cross-connections (same legend as in Figure 2)

\section{B. Solution Qualities and Characteristics}

Optimality gaps were all smaller than $1 \%$, sometimes even of the order of $0.1 \%$ or less. In addition, the computing times required to obtain such high quality solutions are smaller than 10 seconds, therefore the proposed solution schemes for solving the two optimization models are highly scalable, taking into account that several improvements could be easily added to reduce further the computing times (e.g., a heuristic for a "warm" start instead of starting from scratch the solution of the linear programs).

\section{Bandwidth Cost under Dynamic Traffic}

The bandwidth variation cost, at time period $t$, is the difference between the overall protection bandwidth cost at time period $t$ and the overall protection bandwidth cost at time period $t-1$. Results are summarized in Figure 2, where time periods are on the horizontal axis, and bandwidth variation cost on the vertical one. We did experiments with two different values for the number of copies of disturbed $p$-cycles (i.e., parameter $\bar{z}$ in the mathematical models), $5 \%$ and $10 \%$ of established $p$-cycle copies for Model 1 , and with $10 \%$ for Model 2. For Model 2, the number of disturbed $p$-cycle copies does not exceed an average of $1.5 \%$ in all traffic scenarios for the two network instances. We observe that the first model gives the best results in term of bandwidth cost. The best quality with respect to the cost is obtained by the first model with $\bar{z}=10 \%$, see [6] for further results. Remind that the 
objective function of the first model is to minimize the overall bandwidth cost. However, even if the objective function of the second model is to minimize the number of added ports at each time, we can see that the bandwidth cost is not very high compared to the first model.

\section{D. p-Cycle stability under dynamic traffic}

We investigate here the stability of the $p$-cycles under dynamic traffic using the number of reconfigured $p$-cycles as a stability indicator. Figure 3 shows the number of reconfigured/added $p$-cycles at each time period. It is evaluated, at time period $t$ with the sum of the number of dismantled $p$-cycles and of the number of added $p$-cycles. In practice, it corresponds to the number of $p$-cycles a network operator would have to set up or tear down at each time period. We can see that Model 2 clearly outperforms Model 1 in terms of the number of reconfigured/added $p$-cycles. Indeed, the average number of reconfigured/added $p$-cycles for the Model 2 varies between 4.3 and 9.2 for the NY network (see Figure 3(b)) and between 6.2 and 8.5 for COST239 network (see Figure $3(\mathrm{a})$ ), while the average number of reconfigured $p$-cycles for the Model 1 with $\bar{z}=10 \%$, varies between 25.4 and 27.4 for NY network and between 24.0 and 27.2 for COST239 network. Thus, with Model 2, the number of reconfigured $p$ cycles can be reduced by $83.1 \%$ in NY network and by $76.1 \%$ in COST239 network, in comparison with Model 1 assuming $\bar{z}=10 \%$ of established $p$-cycle copies.

\section{E. Pre-cross-connection updates}

The second indicator of stability that we now investigate is the number of pre-cross-connection updates at each time period. Figure 4 shows that the average number of pre-crossconnection updates for Model 2 varies between 26.5 and 43.1 for NY network (see Figure 4(b)) and between 30.5 and 43.1 for COST239 network (see Figure 4(a)), while the average number of pre-cross-connection updates for Model 1 with $\bar{z}$ $=10 \%$ of established $p$-cycle copies, varies between 267.2 and 282.6 for NY network and between 212.5 and 235.9 for COST239 network. Thus, with Model 2, the number of precross-connection updates can be reduced by $90.1 \%$ in NY network and by $87.1 \%$ in COST239 network, compared to Model 1 with $\bar{z}=10 \%$. It is a huge difference.

If we turn our attention to the results in section IV-D, we can see that the reduction made by Model 2 in terms of number of reconfigured/added $p$-cycles compared to Model 1 varies between $65.7 \%$ and $83.1 \%$ in NY network and between 64.6 $\%$ and $76.1 \%$ in COST239 network, while the reduction in terms of the number of pre-cross-connection updates varies between $84.2 \%$ and $90.1 \%$ in NY network and between $80.0 \%$ and $87.1 \%$ in COST239 network. The reduction in terms of the number of pre-cross-connection updates is therefore very significant compared to the reduction in terms of number of $p$-cycle copies as: (i) the average length of the reconfigured/added $p$-cycles is greater in Model 1 and (ii) precross-connections of the reconfigured/added $p$-cycles in Model
2 are saved ones from the previously established $p$-cycles that are re-used in some of the newly established $p$-cycles.

\section{CONCLUSION}

In this paper, we revisited the issue related to the controversial issue of the stability of $p$-cycles under dynamic traffic. While we first investigated this issue in [6] and found out that the stability was moderate under the classical objective of the minimum spare capacity protection design, we showed in this paper that we indeed observe a significant stability in terms of the need of setting new OXC pre-cross-connections with the new objective. In other words, even if all $p$-cycles are not preserved from one time period to the next, only few new OXC pre-cross-connections need to be set. In addition, we designed and provided highly scalable optimization models to conduct this analysis and therefore to perform the $p$-cycle updates under dynamic traffic.

\section{ACKNOWLEDGMENT}

The first author has been supported by a Concordia University Research Chair (Tier I) and by an NSERC (Natural Sciences and Engineering Research Council of Canada) grant.

\section{REFERENCES}

[1] W. D. Grover and D. Stamatelakis, "Cycle-oriented distributed preconfiguration: Ring-like speed with mesh-like capacity for self-planning network restoration," in IEEE International Conference on Communications (ICC 1998), June 1998, pp. 537-543.

[2] W. Zhong and Z. Zhang, " $p$-cycles-based dynamic protection provisioning in optical WDM networks," IEICE Transactions on Communications, vol. 88, no. 5, pp. 1921-1926, 2005.

[3] L. Ruan and F. Tang, "Dynamic establishment of restorable connections using $p$-cycle protection in WDM networks," in International Conference on Broadband Networks, vol. 1, October 2005, pp. 137 - 144.

[4] A. Eshoul and H. Mouftah, "Survivability approaches using $p$-cycles in WDM mesh networks under static traffic," IEEE/ACM Transactions on Networking (TON), vol. 17, no. 2, pp. 671-683, April 2009.

[5] A. Grue, W. Grover, M. Clouqueur, D. Schupke, J.Doucette, B. Forst, D. Onguetou, and D. Baloukov, "Comparative study of fully pre-crossconnected protection architectures for transparent optical networks," in Workshop on Design of Reliable Communication Networks - DRCN, 2007, pp. $1-8$.

[6] A. Metnani and B. Jaumard, "Directed $p$-cycle protection in dynamic WDM networks," in IEEE International Workshop on Reliable Networks Design and Modeling - RNDM, St Petersburg, Russia, October 2009, pp. $1-6$.

[7] W. Grover, "Globally optimal distributed synchronous batch reconfiguration for efficient hazard-free dynamic provisioning: How an entire network can " think globally and act locally"?" in Proceedings of IEEE/VDE Workshop on Design of Reliable Communication Networks - DRCN, October 2007, pp. 1-8.

[8] J. Doucette, D. He, W. Grover, and O. Yang, "Algorithmic approaches for efficient enumeration of candidate $p$-cycles and capacitated $p$-cycle network design," in Proceedings of IEEE/VDE Workshop on Design of Reliable Communication Networks - DRCN, October 2003, pp. 212-220.

[9] B. Jaumard, C. Rocha, D. Baloukov, and W. D. Grover, "A column generation approach for design of networks using path-protecting $p$ cycles," in Proceedings of IEEE/VDE Workshop on Design of Reliable Communication Networks - DRCN, October 2007.

[10] C. Rocha and B. Jaumard, "Revisiting $p$-cycles / FIPP $p$-cycles vs. shared link / path protection," in International Conference on Computer Communications and Networks - ICCCN, 2008, pp. 1-6.

[11] D. Brélaz, "New methods to color the vertices of a graph," Communications of the ACM, vol. 22, no. 4, 1979.

[12] P. Batchelor et al., "Ultra high-capacity optical transmission networks: Final report of action COST 239," Faculty of Electrical Engineering and Computing, University of Zagreb, Tech. Rep., 1999. 